\title{
Dust production by metal-poor stars
}

\author{
lain McDonald* \\ University of Manchester \\ E-mail: mcdonaldejb.man.ac.uk
}

I summarise work carried out on the differences in dust production by metal-poor stars, compared to canonical expectations from the Galaxy. Our conclusions are as follows. Dust may be more efficiently produced by low-mass, metal-poor stars due to their faster RGB evolution. The onset of dust production appears correlated with pulsation, which begins at slightly higher luminosities for metal-poor stars. The dust condensation sequence appears little changed for metal-poor carbon stars. Metal-poor oxygen-rich stars show marked changes in dust condensation products, with a dramatic shift occurring for globular cluster stars below $[\mathrm{Fe} / \mathrm{H}]=-1$. A lack of oxygen-rich AGB stars in globular clusters much below this metallicity currently prevents further analysis. We consider the mass-loss rate to be set by magneto-acoustic or pulsation processes on the stellar surface. Dust-driving may only become important at the highest dust-production rates where its primary role is to set the wind velocity. Future work should concentrate on obtaining gas massloss rates and wind velocities for AGB stars across a variety of dust-production rates, chemistries and metallicities to conclusively determine the role of dust in driving stellar winds.

The Life Cycle of Dust in the Universe: Observations, Theory, and Laboratory Experiments 18-22 November, 2013

Taipei, Taiwan

* Speaker. 


\section{Introduction}

Metal-poor stars represent the first steps in the transition from a $\mathrm{H}-\mathrm{He}$ Universe to the dusty Universe of today. Study of these stars helps trace this journey, yet only the lowest-mass metal-poor stars remain extant, observable in nearby dwarf galaxies and globular clusters. Yet these stars open a new parameter space, where we can test metallicity-dependent phenomena such as dust-driving of winds and carbon star production. In this review, we summarise our recent work on the subject to answer three questions of importance to the field.

\section{Is dust-production efficiency constant?}

Stellar physics tells us that low mass stars evolve slowly enough and have thin enough atmospheres that mass loss before the thermally pulsating asymptotic giant branch (TP-AGB) phase is important. Only in this phase is dust expected to be produced. Since metal-poor stars of a given mass evolve faster, we can expect less mass to be lost before the TP-AGB. More mass should therefore be left to produce dust, and the AGB should extend to higher luminosities in metal-poor systems [1]. But the question remains of how significant this is.

To investigate, we probe globular clusters, which represent a relatively small spread in mass (and other parameters), but exhibit a broad range of metallicities. Mass loss can be measured in three ways: (1) by measuring the difference in mass between stars at different stages of evolution using differences in surface gravity (e.g. [2]) or astro-seismological properties (e.g. [3]); (2) by measuring the mass of horizontal branch stars and comparing them to their initial masses (e.g. [1]); or (3) by directly measuring the mass loss rate through chromospheric tracers (e.g. [4-6]) or dusty winds (e.g. [7-10]).

Mass-loss rates acquired by method (3) make many assumptions, and represent instantaneous snapshots of very dynamic systems [7]. Method (1) is not widely used, due to the complexity in data collection and analysis. An analysis of 54 globular clusters using method (2) will be published soon. Here, we parameterise red giant branch (RGB) mass-loss efficiency following [11] and [12]. This efficiency is remarkably constant, varying by only a few percent at most over a factor of 100 in metallicity ( $\eta_{\text {Reimers }} \approx 0.55$ on average). We show that while the mass lost during the TP-AGB phase of stellar evolution appears roughly similar for the most metal-rich and metal-poor clusters, this is defined partly by the difference in initial mass of those stars. We can expect, for a given mass, that metal-poor stars will lose more mass via a dusty wind than an otherwise-identical metal-rich star, and reach higher luminosities on the AGB.

\section{When does dust production start, and is it linked to pulsation?}

The above argument does not take into account observations of dust-producing stars in globular clusters, which help us define the onset of dust production and its cessation at the AGB tip. Based on our soon-to-be-published modelling, we expect that the AGB tip in metal-rich globular clusters should lie below the RGB tip $\left(\approx 2500 \mathrm{~L}_{\odot}\right)$, rising to over $3000 \mathrm{~L}_{\odot}$ in metal-poor systems (where the RGB tip is at $\approx 1600 \mathrm{~L}_{\odot}$ ). The observations show the opposite, with well-populated AGBs in metal-rich clusters and a paucity of bright, dusty AGB stars in metal-poor clusters [13, 14], possibly indicating an incomplete treatment of mass loss on the upper AGB. 
Typically, one expects that mass loss may be enhanced either by stellar pulsation or radiation pressure on dust (e.g. [15]). Momentum absorbed by dust must be sufficient to counteract gravity before mass loss can be enhanced, but dust must also drag the surrounding gas with it to escape, hence we should expect a strong metallicity dependence on radiation-driven mass loss. By comparison, stellar pulsation should be much less metallicity dependent, only depending on the depth of the hydrogen ionisation layer (for $\kappa$-mechanism pulsation).

Observationally, one finds that the onset of dust production has only a weak metallicity dependence, depending mostly on when strong pulsations occur. Analysis of the Hipparcos dataset [16] identified 62 dusty giants within $\sim 300 \mathrm{pc}$, almost all of which have entries in variable star catalogues. In globular clusters, we find that stars with circumstellar dust production are almost invariably those with strong pulsation ( $\delta V \gtrsim 0.1 \mathrm{mag}$; [13,9]). This criterion is found at slightly brighter luminosities for metal-poor stars, probably because they are slightly hotter. Pulsation seems to be required to generate a dusty wind, suggesting that pulsation sets the mass-loss rate, while dust driving sets the terminal wind velocity (as in [17]).

Our observations in the Sgr dSph have emphasised that the detection of long-period variability in stars is simply down to the sensitivity of observations. However, dust production probably begins at a similar pulsation amplitude $[18,19]$. There is no difference between the amplitude of pulsation nor the surface characteristics of RGB and AGB stars, raising the question of why the RGB stars do not appear to be producing dust in similar quantities to AGB stars of the same luminosity.

\section{Does the dust condensation sequence change at low metallicity?}

The dust condensation sequence may change at low metallicity, due to the change in chemical equilibrium and change in the $[\alpha / \mathrm{Fe}]$ ratio of metal-poor stars. Research on carbon-rich stars shows little change in the dust condensation sequence. The dominant dust product of carbon stars is thought to be carbon, in various forms of lattice structure and hydrogenation from nanodiamonds to polycyclic aromatic hydrocarbons to hydrogenated amorphous carbon. Carbon stars produce their own carbon, so the resulting dust condensation sequence shows only minor changes in secondary minerals such as $\mathrm{SiC}$, and the production of hydrogenated molecules like $\mathrm{C}_{2} \mathrm{H}_{2}$ [20].

Oxygen-rich stars, however, show considerable differences. Dust chemistry here is dominated by the stars' initial abundances. The dominant dust product of oxygen-rich stars is typically silicate dust. [21] has shown that the relative oxygenation of crystalline silicates increases as one goes to lower metallicity, with enstatites becoming less common than forsterites in the Magellanic Clouds, compared to the Galaxy. This is also seen in globular cluster stars, where silicate features are also seen to disappear in stars below $[\mathrm{Fe} / \mathrm{H}] \sim-1$ [9]. That is not to say that dust production stops, as stars clearly have (featureless) excess flux at infrared wavelengths.

We have argued that this excess is attributable to metallic iron dust [8]. Other talks at this conference have concentrated on the missing iron problem, whereby iron is depleted from the gas phase in various circumstellar and interstellar environments. Yet metallic iron is only expected to condense after silicates, and the reasons silicates are not seen are yet to be explained. Other explanations for the large excess include amorphous carbon [22] and large-grained silicates [23, 24], however these may be difficult to create around metal-poor evolved stars. 


\section{Future work}

Our observations suggest that magneto-acoustic process and pulsation are more likely to drive winds from oxygen-rich stars, with radiation pressure on dust only becoming significant when winds become optically thick. They suggest a change of condensation sequence in metal-poor stars, whereby transparent iron-free silicates are gradually replaced by opaque iron dust over a transition near $[\mathrm{Fe} / \mathrm{H}]=-1$. We therefore hypothesize: (1) that there should be little change in outflow velocity for stars of similar mass-loss rates but different metallicities, as dust does not drive the wind; and (2) a polarisation signature should be observable from iron dust around metalpoor evolved stars (cf. [25]). These hypotheses are difficult to test accurately in the field, due to the distant nature of the globular cluster stars we have used to test them. Initial ALMA results [?] show that it may be difficult to observe $\mathrm{CO}(2-1)$ in metal-poor stars in globular clusters, requiring long integration times. A closer target is needed.

In our analysis of the Hipparcos stars, we investigated the nearby star EU Del [16]. This star is thought to be metal-poor [26], though an accurate metallicity is difficult to determine due to the strong molecular features. We presented literature photometry, including an IRAS spectrum, which shows it to have infrared excess, but no silicate features. It may therefore be analogous to the globular cluster AGB stars we have been investigating. Recently collected APEX data [?] observed the $\mathrm{CO}(3-2)$ transition of this star. Initial results show a typical wind velocity, provided initial confirmation our first hypothesis.

Finally, we note that a considerable disparity exists between the mass-loss rates derived for globular cluster AGB stars (and EU Del) and that predicted by evolutionary theory [?]. Mass-loss rates for AGB stars may be significantly over-estimated. While a number of factors affect mass-loss rates derived from infrared spectra, the most important is likely to be the stellar outflow velocity [7]. If we are to understand the role of stellar mass loss in galactic ecology, it is vital we measure gas mass-loss rates and wind velocities of metal-poor stars. The only instrument that is capable of obtaining a statistically meaningful sample of such data is ALMA, and we look forward to results from it.

\section{References}

[1] R. G. Gratton, et al., A\&A, 517, A81

[2] I. McDonald, C. I. Johnson, and A. A. Zijlstra, 2011, MNRSA, 416, L6

[3] A. Miglio, et al., 2012, MNRAS, 419, 2077

[4] A. K. Dupree, L. Hartmann, and E. H. Avrett, 1984, ApJ, 281, L84

[5] I. McDonald and J. Th. van Loon, 2007, A\&A, 476, 1261

[6] S. Mészáros, E. H. Avrett, and A. K. Dupree, 2009, AJ, 138, 615

[7] I. McDonald, et al., 2011, ApJ, 730, 71

[8] I. McDonald, et al., 2010, ApJ, 717, L92

[9] I. McDonald, et al., 2011, MNRAS, 417, 20

[10] I. McDonald, et al., 2009, MNRAS, 394, 831 
[11] D. Reimers., 1975, Mem. Soc. Roy. Sci. Liège, 8, 369

[12] K.-P. Schröder and M. Cuntz, 2005, ApJ, 630, L73

[13] I. McDonald, et al., 2011, ApJS, 193, 23

[14] I. McDonald, J. T. van Loon, A. K. Dupree, and M. L. Boyer, 2010, MNRAS, 405, 1711

[15] P. Woitke., 2006, A\&A, 460, L9

[16] I. McDonald, A. A. Zijlstra, and M. L. Boyer, 2012, MNRAS, 427, 343

[17] J. M. Winters, et al., 2000, A\&A, 361, 641

[18] I. McDonald, et al., 2014, MNRAS, 439, 2618

[19] I. McDonald, et al., 2013, MNRAS, 436, 413

[20] G. C. Sloan, et al., 2012, ApJ, 752, 140

[21] O. C. Jones, et al., 2012, MNRAS, 427, 3209

[22] S. Höfner and A. C. Andersen. 2007, A\&A, 465, L39

[23] S. Höfner, 2008, A\&A, 491, L1

[24] B. R. M. Norris, et al., 2012, Nature, 484, 220

[25] B. T. Draine and A. Lazarian, 1999, ApJ, 512, 740

[26] Y. Wu, et al., 2011, A\&A, 525, A71

[27] I. McDonald, et al., 2014a, in prep.

[28] I. McDonald, et al., 2014b, in prep.

[29] I. McDonald, et al., 2014, submitted MNRAS 